\title{
Gravitational radiation fields in teleparallel equivalent of general relativity and their energies *
}

\author{
Gamal G.L. Nashed ${ }^{\dagger}$ \\ Centre for Theoretical Physics, The British University in Egypt, El-Sherouk City, \\ Misr - Ismalia Desert Road, Postal No. 11837, P.O. Box 43, Egypt. \\ e-mail: nashed@bue.edu.eg
}

\begin{abstract}
We derive two new retarded solutions in the teleparallel theory equivalent to general relativity (TEGR). One of these solutions gives a divergent energy. Therefore, we used the regularized expression of the gravitational energy-momentum tensor, which is a coordinate dependent. A detailed analysis of the loss of the mass of Bondi space-time is carried out using the flux of the gravitational energy-momentum.
\end{abstract}

\section{Introduction}

The linearized theory has been developed extensively, however, it seems doubtful if its results can be fully trusted [1]. The non-linearity of the gravitational field is the most 'characteristic properties and some of the crucial properties of the field show themselves only 'through the non-linear terms. Additionally, it is never clear whether solutions derived from the linearization corresponds to exact solutions [1]. In spite that we have a good deal about ' exact plane and cylindrical wave solutions, it is doughtily whether such solutions display the characteristics of physically significant waves [2]. Einstein's General relativity (GR) theory is a complete one however, it may not give sensible solutions for situations too far removed from what is physically reasonable. The most simplest field due to a finite source which can be obtained from GR is the spherically symmetric solution, however, Birkhoff's theorem shows that such solution must be static. For this reason, one cannot be truly speak about spherically symmetric waves and thus any description of radiation from a finite system must necessary involve three coordinate significantly. This makes the mathematical calculations more complicated and thus we have to make use of methods of approximation.

Two well established formulas of gravitational energy are the ADM (Arnowitt-DeserMisner) energy that corresponds to the total energy [3] and the Bondi energy [2] which describes the mass of radiating systems in asymptotic flat space-times. The loss of energy

PACS numbers: 04.20.Cv, 04.20.Fy, 04.50.-h

Keywords: teleparallel equivalent of general relativity, energy-momentum tensor, Bondi mass, gravitational radiation

Mtathematics Department, Faculty of Science, Ain Shams University, Cairo, Egypt 
of the total mass of a source that radiates gravitational waves is main feature of the Bondi energy [2], i.e.,

$$
\frac{d E}{d t}=-\left(\frac{d c_{0}}{d t}\right)^{2},
$$

where $c_{0}$ is the news function. Eq. (1) shows that Bondi energy is related to the loss of the total mass of a source that radiates gravitational waves. The loss of the total mass is given in terms of the minus news function squared which means that the mass of the source can decrease.

Teleparallel theories are interesting for several reasons: first of all, general relativity (GR) can be viewed as a particular theory of teleparallelism and, thus, teleparallelism could be considered at the very least as a different point of view that can lead to the same results [4]. Second, in this framework, one can define an energy-momentum tensor for the gravitational field that is a true tensor under all general coordinate transformations. This is the reason why teleparallelism was reconsidered by Møller when he was studying the problem of defining an energy-momentum tensor for the gravitational field [5]. The idea was taken over by Pellegrini and Plebański that constructed the general Lagrangian for these theories [6]. The third reason why these theories are interesting is that they can be seen as gauge theories of the translation group (not the full Poincare group) and, thus, they give an alternative interpretation of GR $[7,8,9,10]$. Therefore, we consider the tetrad theory of gravitation in this work.

It is the aim of the present work to study the variation of the gravitational energy and the corresponding total flux of energy at spacelike infinity within the framework of TEGR. To do so we derive the solution given by Bondi [2] in the framework of TEGR and then compute the total mass using the energy-momentum tensor. Also we compute the loss of mass using the flux of the gravitational energy-momentum tensor [11]. In $\S 2$, we briefly review the TEGR theory for gravitational field and its derivation of the field equations. A summary of the derivation of energy and total fluxes of the gravitational energy-momentum are also given in $\S 2$. In $\S 3$, we study the most general tetrad field which contains 16 unknown functions. Two new solutions are also given in $\S 3$. Then, we calculate the total mass of these solutions using the regularized expression in $\S 4$. Using the flux of the gravitational energy-momentum we also calculate the loss of mass in $\S 4$. Final section is devoted for the main results.

\section{The continuity equation and the fluxes of the gravitational and matter fields}

In a space-time with absolute parallelism the parallel vector fields $e_{a}{ }^{\mu}$ define the nonsymmetric affine connection

$$
\Gamma_{\mu \nu}^{\lambda} \stackrel{\text { def. }}{=} e_{a}^{\lambda} e^{a}{ }_{\mu, \nu}
$$

where $e_{a \mu, \nu}=\partial_{\nu} e_{a \mu}{ }^{*}$. The curvature tensor defined by $\Gamma_{\mu \nu}^{\lambda}$, given by Eq. (2), is identically vanishing. The metric tensor $g_{\mu \nu}$ is defined by

$$
g_{\mu \nu} \stackrel{\text { def. }}{=} \eta_{a b} e^{a}{ }_{\mu} e^{b}{ }_{\nu}
$$

${ }^{*}$ space-time indices $\mu, \nu, \cdots$ and $\mathrm{SO}(3,1)$ indices $\mathrm{a}, \mathrm{b} \cdots$ run from 0 to 3 . Time and space indices are indicated to $\mu=0, i$, and $a=(0),(i)$. 
with $\eta_{a b}=(-1,+1,+1,+1)$ is the metric of Minkowski space-time.

The Lagrangian density for the gravitational field in the TEGR, in the presence of matter fields, is given $b^{\dagger}[11]$

$$
\mathcal{L}_{G}=e L_{G}=-\frac{e}{16 \pi}\left(\frac{T^{a b c} T_{a b c}}{4}+\frac{T^{a b c} T_{b a c}}{2}-T^{a} T_{a}\right)-L_{m}=-\frac{e}{16 \pi} \Sigma^{a b c} T_{a b c}-L_{m}
$$

where $e=\operatorname{det}\left(e^{a}{ }_{\mu}\right)$. The tensor $\Sigma^{a b c}$ is defined by

$$
\Sigma^{a b c} \stackrel{\text { def. }}{=} \frac{1}{4}\left(T^{a b c}+T^{b a c}-T^{c a b}\right)+\frac{1}{2}\left(\eta^{a c} T^{b}-\eta^{a b} T^{c}\right)
$$

$T^{a b c}$ and $T^{a}$ are the torsion tensor and the basic vector field defined by

$$
T_{\mu \nu}^{a} \stackrel{\text { def. }}{=} e_{\lambda}^{a} T_{\mu \nu}^{\lambda}=\partial_{\mu} e^{a}{ }_{\nu}-\partial_{\nu} e^{a}{ }_{\mu}, \quad T_{b c}^{a} \stackrel{\text { def. }}{=} e_{b}{ }^{\mu} e_{c}{ }^{\nu} T_{\mu \nu}^{a} \quad \quad T^{a} \stackrel{\text { def. }}{=} T_{b}^{b}{ }_{b}^{a} .
$$

The quadratic combination $\Sigma^{a b c} T_{a b c}$ is proportional to the scalar curvature $R(e)$, except for a total divergence term [11]. $L_{m}$ represents the Lagrangian density for matter fields.

The gravitational field equations described by $L_{G}$ are the following

$$
e_{a \lambda} e_{b \mu} \partial_{\nu}\left(e \Sigma^{b \lambda \nu}\right)-e\left(\Sigma_{a}^{b \nu} T_{b \nu \mu}-\frac{1}{4} e_{a \mu} T_{b c d} \Sigma^{b c d}\right)=\frac{1}{2} \kappa e T_{a \mu}
$$

where

$$
\frac{\delta L_{m}}{\delta e^{a \mu}} \equiv e T_{a \mu}
$$

It is possible to prove by explicit calculations that the left hand side of the symmetric field equations $(7)$ is exactly given by [11]

$$
\frac{e}{2}\left[R_{a \mu}(e)-\frac{1}{2} e_{a \mu} R(e)\right]
$$

Multiplication of Eq. (7) by the appropriate inverse tetrad fields yields it to have the form $[11,12]$

$$
\partial_{\nu}\left(-e \Sigma^{a \lambda \nu}\right)=-\frac{e e^{a \mu}}{4}\left(4 \Sigma^{b \lambda \nu} T_{b \nu \mu}-\delta^{\lambda}{ }_{\mu} \Sigma^{b d c} T_{b c d}\right)-4 \pi e_{\mu}^{a} T^{\lambda \mu} .
$$

By restricting the space-time index $\lambda$ to assume only spatial values then Eq. (8) takes the form [11]

$$
\partial_{0}\left(e \Sigma^{a 0 j}\right)+\partial_{k}\left(e \Sigma^{a k j}\right)=-\frac{e e^{a \mu}}{4}\left(4 \Sigma^{b c j} T_{b c \mu}-\delta^{j}{ }_{\mu} \Sigma^{b c d} T_{b c d}\right)-4 \pi e e^{a}{ }_{\mu} T^{j \mu} .
$$

Note that the last two indices of $\Sigma^{a b c}$ and $T^{a b c}$ are anti-symmetric. Taking the divergence of Eq. (9) with respect to j yields

\footnotetext{
${ }^{\dagger}$ Throughout this paper we use the relativistic units , $c=G=1$ and $\kappa=8 \pi$.
} 


$$
-\partial_{0} \partial_{j}\left(-\frac{1}{4 \pi} e \Sigma^{a 0 j}\right)=-\frac{1}{16 \pi} \partial_{j}\left[e e^{a \mu}\left(4 \Sigma^{b c j} T_{b c \mu}-\delta^{j}{ }_{\mu} \Sigma^{b c d} T_{b c d}\right)-16 \pi\left(e e_{\mu}^{a} T^{j \mu}\right)\right] .
$$

The Hamiltonian formulation of TEGR is obtained by establishing the phase space variables. The Lagrangian density does not contain the time derivative of the tetrad component $e_{a 0}$. Therefore, this quantity will arise as a Lagrange multiplier [13]. The momentum canonically conjugated to $e_{a i}$ is given by $\Pi^{a i}=\delta L / \delta \dot{e}_{a i}$. The Hamiltonian formulation is obtained by rewriting the Lagrangian density in the form $L=p \dot{q}-H$, in terms of $e_{a i}, \Pi^{a i}$ and the Lagrange multipliers. The Legendre transformation can be successfully carried out and the final form of the Hamiltonian density has the form [14]

$$
H=e_{a 0} C^{a}+\alpha_{i k} \Gamma^{i k}+\beta_{k} \Gamma^{k}
$$

plus a surface term. Here $\alpha_{i k}$ and $\beta_{k}$ are Lagrange multipliers that are identified as

$$
\alpha_{i k}=\frac{1}{2}\left(T_{i 0 k}+T_{k 0 i}\right) \quad \text { and } \quad \beta_{k}=T_{00 k}
$$

where $C^{a}, \Gamma^{i k}$ and $\Gamma^{k}$ are first class constraints.

The constraint $C^{a}$ is written as $C^{a}=-\partial_{i} \Pi^{a i}+h^{a}$, where $h^{a}$ is an intricate expression of the field variables. The integral form of the constraint equation $C^{a}=0$ motivates the definition of the gravitational energy-momentum $P^{a}$ four-vector [11]

$$
P^{a}=-\int_{V} d^{3} x \partial_{i} \Pi^{a i}
$$

where $V$ is an arbitrary volume of the three-dimensional space. In the configuration space we have

$$
\begin{gathered}
\Pi^{a i}=-4 \kappa \sqrt{-g} \Sigma^{a 0 i} \quad \text { with } \quad \partial_{\nu}\left(\sqrt{-g} \Sigma^{a \lambda \nu}\right)=\frac{1}{4 \kappa} \sqrt{-g} e^{a}{ }_{\mu}\left(t^{\lambda \mu}+T^{\lambda \mu}\right) \text { where } \\
t^{\lambda \mu}=\kappa\left(4 \Sigma^{b c \lambda} T_{b c}{ }^{\mu}-g^{\lambda \mu} \Sigma^{b c d} T_{b c d}\right) .
\end{gathered}
$$

By integrating Eq. (10) on a volume $V$ of the three-dimensional space we get [11]

$$
\frac{d}{d t}\left[-\int_{v} d^{3} x \partial_{j} \Pi^{a j}\right]=-\phi_{g}{ }^{a}-\phi_{m}{ }^{a},
$$

where

$$
\phi_{g}{ }^{a}=\frac{\kappa}{2} \int_{S} d S_{j}\left[e e^{a \mu}\left(4 \Sigma^{b c j} T_{b c \mu}-\delta_{\mu}{ }^{j} \Sigma^{b c d} T_{b c d}\right)\right],
$$

is the $a$ component of the gravitational energy-momentum flux and

$$
\phi_{m}{ }^{a}=\int_{S} d S_{j}\left(e e^{a}{ }_{\mu} T^{j \mu}\right)
$$


is the $a$ component of the matter energy-momentum flux. $S$ represent the spatial boundary of the volume $V$. Therefore the loss of the gravitational energy $P^{(0)}=E$ is governed by the equation

$$
\frac{d E}{d t}=-\phi_{g}{ }^{0}-\phi_{m}{ }^{0} .
$$

Maluf and Faria have shown that $\phi_{g}{ }^{0}$ played a major role to the Bondi's radiating spacetime, since it yields the well known expression for the gravitational energy loss, while $\phi_{m}{ }^{0}$ is related to the Vaidya's space-time [15].

\section{Bondi space-times in TEGR and their associated energies}

In this section we are going to use the most general tetrad field which contains 16 unknown functions. Let us write it in the coordinate $(t, r, \theta, \phi)$ by

$$
\left(e^{a}{ }_{\mu}\right)=\left(\begin{array}{llll}
A_{1} & A_{2} & A_{3} & A_{4} \\
B_{1} & B_{2} & B_{3} & B_{4} \\
F_{1} & F_{2} & F_{3} & F_{4} \\
H_{1} & H_{2} & H_{3} & H_{4}
\end{array}\right),
$$

where $A_{i}, B_{i}, F_{i}, H_{i}, i=1 \cdots 4$ are unknown functions of $r, \theta, \phi$ and $t$. Applying Eq. (19) to the field equations (7) we obtained a very lengthy and tedious system of partial differential equations. We are going to write a special solutions to these system.

First solution:

The 16 unknown functions in this solution have the form

$$
\begin{aligned}
& A_{1}=-e^{\beta} \sqrt{\frac{V}{r}}, \quad A_{2}=-e^{\beta} \sqrt{\frac{r}{V}}, \quad A_{3}=0, \quad A_{4}=0, \quad B_{1}=U r e^{\gamma} \cos \theta \cos \phi, \\
& B_{2}=-e^{\beta} \sqrt{\frac{r}{V}} \sin \theta \cos \phi, \quad B_{3}=-r e^{\gamma} \cos \theta \cos \phi, \quad B_{4}=-r e^{-\gamma} \sin \theta \sin \phi, \\
& F_{1}=U r e^{\gamma} \cos \theta \sin \phi, \quad F_{2}=-e^{\beta} \sqrt{\frac{r}{V}} \sin \theta \sin \phi, \quad F_{3}=-r e^{\gamma} \cos \theta \sin \phi, \\
& F_{4}=r e^{-\gamma} \sin \theta \cos \phi, \quad H_{1}=-U r e^{\gamma} \sin \theta, \quad H_{2}=-e^{\beta} \sqrt{\frac{r}{V}} \cos \theta, \\
& H_{3}=r e^{\gamma} \sin \theta, \quad H_{4}=0 .
\end{aligned}
$$

The functions $\beta, \gamma, V$ and $U$ appear in Eq. (20) have the following asymptotic behavior up to $O\left(\frac{1}{r^{2}}\right)$

$$
\begin{aligned}
& \beta=-\frac{\left(c^{2}(u, \theta)+d^{2}(u, \theta)\right)}{4 r^{2}}+O\left(\frac{1}{r^{4}}\right), \quad \gamma=\frac{c(u, \theta)}{r}+O\left(\frac{1}{r^{3}}\right), \\
& U=-\frac{l(u, \theta)}{r^{2}}+O\left(\frac{1}{r^{3}}\right), \quad V=r-2 M(u, \theta)-\frac{l_{1}(u, \theta)}{r},
\end{aligned}
$$


where the time $t$ is defined as $t=u+r$ which is the retarded time. The quantities $l(u, \theta)$ and $l_{1}(u, \theta)$ are defined as

$$
\begin{aligned}
l(u, \theta)= & \frac{\partial c(u, \theta)}{\partial \theta}+2 c(u, \theta) \cot \theta \\
l_{1}(u, \theta)= & \frac{\partial d(u, \theta)}{\partial \theta}+d(u, \theta) \cos \theta-\left(\frac{\partial c(u, \theta)}{\partial \theta}\right)^{2}-4 c(u, \theta)\left(\frac{\partial c(u, \theta)}{\partial \theta}\right) \cot \theta \\
& -\frac{1}{2} c^{2}(u, \theta)\left(1+8 \cot ^{2} \theta\right)
\end{aligned}
$$

with $M(u, \theta), c(u, \theta)$ and $d(u, \theta)$ are the mass aspect, the news function and the dipole aspect respectively. Solution given by Eq. (20) with Eqs. (21) and (22) satisfies the field equations (7) up to $O\left(\frac{1}{r^{2}}\right)$. The metric associated with solution given by Eq. (20) has the form

$$
\begin{aligned}
d s^{2}= & -\left(\frac{V}{r} e^{2 \beta}-U^{2} r^{2} e^{2 \gamma}\right) d u^{2}-2 e^{2 \beta} d u d r \\
& -2 U r^{2} e^{2 \gamma} d u d \theta+r^{2}\left(e^{2 \gamma} d \theta^{2}+e^{2 \gamma} \sin ^{2} \theta d \phi^{2}\right),
\end{aligned}
$$

which is the Bondi space-time [11].

Second solution:

The 16 unknown functions in this case have the form

$$
\begin{aligned}
& A_{1}=\sqrt{\frac{V e^{2 \beta}}{r}+r^{2} e^{2 \gamma} U^{2} \cosh 2 \delta+r^{2} e^{-2 \gamma} W^{2} \cosh 2 \delta+2 r^{2} W U \sinh 2 \delta},
\end{aligned}
$$

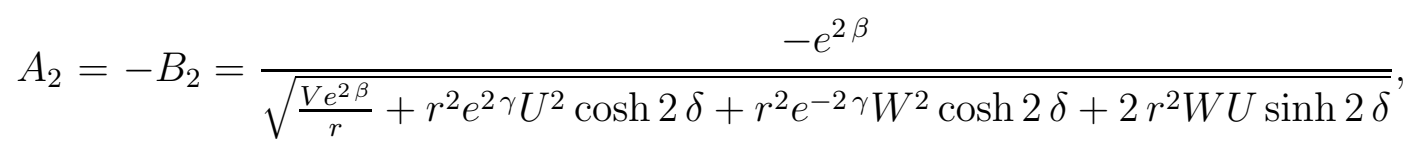

$$
\begin{aligned}
& A_{3}=-B_{3}=\frac{-r^{2}\left(e^{2 \gamma} U \cosh 2 \delta+W \sinh 2 \delta\right)}{\sqrt{\frac{V e^{2 \beta}}{r}+r^{2} e^{2} \gamma U^{2} \cosh 2 \delta+r^{2} e^{-2 \gamma} W^{2} \cosh 2 \delta+2 r^{2} W U \sinh 2 \delta}}, \\
& A_{4}=-B_{4}=\frac{-r^{2} \sin \theta\left(e^{-2 \gamma} W \cosh 2 \delta+U \sinh 2 \delta\right)}{\sqrt{\frac{V e^{2 \beta}}{r}+r^{2} e^{2 \gamma} U^{2} \cosh 2 \delta+r^{2} e^{-2 \gamma} W^{2} \cosh 2 \delta+2 r^{2} W U \sinh 2 \delta}}, \\
& B_{1}=F_{1}=F_{2}=H_{1}=H_{2}=H_{3}=0, \quad F_{3}=r e^{\gamma} \sqrt{-\cosh 2 \delta}, \\
& F_{4}=-\frac{r \sinh 2 \delta \sin \theta}{e^{\gamma} \sqrt{-\cosh 2 \delta}}, \quad H_{4}=\sqrt{-r^{2} e^{-2 \gamma} \cosh 2 \delta \sin ^{2} \theta+\frac{r^{2} \sinh ^{2} 2 \delta \sin ^{2} \theta}{e^{2 \gamma} \cosh 2 \delta}} .
\end{aligned}
$$

The functions $\beta, \gamma, V, U, W$ and $\delta$ appear in Eq. (24) have the following asymptotic behavior up to $O\left(\frac{1}{r^{2}}\right)$

$$
\begin{aligned}
& \beta=-\frac{\left(c^{2}(u, \theta)+d^{2}(u, \theta)\right)}{4 r^{2}}+O\left(\frac{1}{r^{4}}\right), \quad \gamma=\frac{c(u, \theta)}{r}+O\left(\frac{1}{r^{3}}\right), \quad \delta=\frac{d(\theta, u)}{r}+O\left(\frac{1}{r^{3}}\right) \\
& U=-\frac{l(u, \theta)}{r^{2}}+O\left(\frac{1}{r^{3}}\right), \quad V=r-2 M(u, \theta)-\frac{l_{1}(u, \theta)}{r}, \quad W=-\frac{l_{2}(u, \theta)}{r^{2}}+O\left(\frac{1}{r^{3}}\right) .
\end{aligned}
$$

The quantities $l(u, \theta)$ and $l_{1}(u, \theta)$ are defined in Eq. (22) and $l_{2}(u, \theta)$ has the form

$$
l_{2}(u, \theta)=\frac{\partial d(u, \theta)}{\partial \theta}+2 d(u, \theta) \cot \theta
$$


The metric associated with solution given by Eq. (24) has the form

$$
\begin{aligned}
d s^{2}= & \left(\frac{V}{r} e^{2 \beta}+r^{2} U^{2} e^{2 \gamma} \cosh 2 \delta+r^{2} W^{2} e^{-2 \gamma} \cosh 2 \delta+2 r^{2} U W \sinh 2 \delta\right) d u^{2}-2 e^{2 \beta} d u d r \\
& -2 r^{2}\left(U e^{2 \gamma} \cosh 2 \delta+W \sinh 2 \delta\right) d u d \theta-2 r^{2} \sin \theta\left(W e^{-2 \gamma} \cosh 2 \delta+U \sinh 2 \delta\right) d u d \phi \\
& +r^{2}\left(e^{2 \gamma} \cosh 2 \delta d \theta^{2}+e^{-2 \gamma} \cosh 2 \delta \sin ^{2} \theta d \phi^{2}+2 \sinh 2 \delta \sin \theta d \theta d \phi\right),
\end{aligned}
$$

which is the Bondi space-time [16]. Solutions (20) and (24) satisfy the field equations (7) up to $O\left(\frac{1}{r^{3}}\right)$.

Calculate the energy associated with solution (20) by calculating the torsion tensor and basic vector*, we get finally up to $O\left(\frac{1}{r^{0}}\right)$

$$
E \cong \frac{1}{2} \int_{0}^{\pi} \sin \theta\{M(u, \theta)-2 r\} d \theta
$$

which depends on the radial coordinate. Therefore, in this case we are going to use the regularized expression for the gravitational energy-momentum [11].

\section{Regularized expression for the gravitational energy-momentum and localization of energy}

For a space-time in which the physical quantities, i.e., $M(u, \theta)$ etc. equal to zero, it is usual to consider a set of tetrad fields such that the torsion $T^{\lambda}{ }_{\mu \nu}=0$ in any coordinate system. However, in general an arbitrary set of tetrad fields do not satisfy $T_{\mu \nu}^{\lambda}=0$. It might be argued, therefore, that the expression for the gravitational energy-momentum (13) is restricted to particular class of tetrad fields, namely, to the class of frames such that $T^{\lambda}{ }_{\mu \nu}=0$. If $E_{a}{ }^{\mu}$ represents the space-time of the tetrad field in which the physical quantities equal zero [12]. To explain this, let us calculate the space-time in which the physical quantities equal zero for the tetrad field of Eq. (19) using (20) which is given by

$$
\left(E_{a}{ }^{\mu}\right)=\left(\begin{array}{cccc}
-1 & -1 & 0 & 0 \\
0 & -\sin \theta \cos \phi & r \cos \theta \cos \phi & -r \sin \theta \sin \phi \\
0 & -\sin \theta \sin \phi & r \cos \theta \sin \phi & r \sin \theta \cos \phi \\
0 & -\cos \theta & -r \sin \theta & 0
\end{array}\right)
$$

Expression (29) yields the following non-vanishing torsion components:

$$
T_{12}^{2}=\frac{2}{r}=T_{13}^{3}
$$

\footnotetext{
${ }^{*}$ The details components of those quantities are given in the appendix.
} 
We will denote $T^{a}{ }_{\mu \nu}(E)=\partial_{\mu} E^{a}{ }_{\nu}-\partial_{\nu} E^{a}{ }_{\mu}$ and $\Pi^{a j}(E)$ as the expression of $\Pi^{a j}$ constructed out of the tetrad $E^{a}{ }_{\mu}$. The regularized form of the gravitational energy-momentum $P^{a}$ is defined by $[12,14]$

$$
P^{a}=-\int_{V} d^{3} x \partial_{k}\left[\Pi^{a k}(e)-\Pi^{a k}(E)\right] .
$$

The reference space-time is determined by tetrad fields $E^{a}{ }_{\mu}$, obtained from $e^{a}{ }_{\mu}$ by requiring the vanishing of the physical parameters like mass, angular-momentum, etc. Eq. (31) can have the form $[14,12]$

$$
P^{a}=-\oint_{S \rightarrow \infty} d S_{k}\left[\Pi^{a k}(e)-\Pi^{a k}(E)\right],
$$

where the surface $S$ is established at spacelike infinity. Eq. (32) transforms as a vector under the global $\mathrm{SO}(3,1)$ group [11].

Let us use Eq. (32) to calculate the gravitational energy of Eq. (20). We need to calculate the quantity

$$
\Sigma^{(0) 01}=e^{(0)}{ }_{0} \Sigma^{001}+e^{(0)}{ }_{a} \Sigma^{a 01} .
$$

Evaluate the above equation we find

$$
\Pi^{(0) 1}(e) \cong \frac{1}{4} \sin \theta\{M(u, \theta)-4 r\} .
$$

The expression of $\Pi^{(0) 1}(E)$ is obtained by just making $M(u, \theta)=0$, in Eq. (33), it is given by

$$
\Pi^{(0) 1}(E) \cong-r \sin \theta .
$$

Using Eqs. (33) and (34) in Eq. (32) we finally get the gravitational energy of the tetrad field of Eq. (19) using solution given by Eq. (20) in the form

$$
P^{(0)} \cong \frac{1}{2} \int_{0}^{\pi} \sin \theta M(u, \theta) d \theta
$$

which is the Bondi energy [2].

The flux of the gravitational energy which is determined by putting $a=(0)$ in Eq. (16) and by making $j=1$, i.e., by integrating over a surface $S$ of constant radius $r$, and requiring $r \rightarrow \infty$. Eq. (16) in such case has the form

$$
\phi_{g}{ }^{(0)}=\frac{\kappa}{2} \int_{S} d S_{1} e\left(4 e^{(0) \mu} \Sigma^{b c 1} T_{b c \mu}-e^{(0) 1} \Sigma^{b c d} T_{b c d}\right) .
$$

Applying Eq (36) to the tetrad field (19) using Eq. (20), we finally get

$$
\phi_{g}{ }^{(0)}=\frac{1}{2} \int_{0}^{\pi} \sin \theta\{c, 0(u, \theta)\}^{2} d \theta,
$$

which is the value of the loss of the gravitational mass of the Bondi space-time [2]. In that case Eq. (1) is satisfied for solution given by Eq. (20). 
Using the regularized form of the energy given by Eq. (32) to calculate the gravitational energy of $\mathrm{Eq}(24)$, we finally get

$$
\Pi^{(0) 1}(e) \cong \frac{1}{4} \sin \theta\{M(u, \theta)-r\}
$$

The expression of $\Pi^{(0) 1}(E)$ is obtained by just making $M(u, \theta)=0$, in Eq. (38), it is given by

$$
\Pi^{(0) 1}(E) \cong-r \sin \theta .
$$

Thus the gravitational energy of Eq. (24) is given by

$$
P^{(0)} \cong \frac{1}{2} \int_{0}^{\pi} \sin \theta M(u, \theta) d \theta
$$

which is the Bondi energy [2].

Repeat the same calculations done for the first solution we finally, get the flux of the gravitational energy of the second solution in the form

$$
\phi_{g}^{(0)}=\frac{1}{2} \int_{0}^{\pi} \sin \theta\left(\{c, 0(u, \theta)\}^{2}+\{d, 0(u, \theta)\}^{2}\right) d \theta
$$

which is the value of the loss of mass of the Bondi space-time $[2,16]$. The flux given by Eq. (41) will coincide with that of Eq. (37) when the function $d(u, \theta)=0$ which means that the function $\delta$ appears in solution (24) must vanish.

\section{Main results and Discussion}

The main results of the present paper are the following:

- We have obtained two new special solutions which satisfy the field equations (7). These solutions reproduce the Bondi space-time $[2,11,16]$.

- We have calculated the energy associated with the first solution and found that it is depends on the radial coordinate. This is due to the fact if the physical quantities, i.e., $M(u, \theta)$ set equal zero we show that the components of the torsion tensor did not vanishing as it should be! Therefore, we have used the regularized expression to recalculate the energy associated with the two solutions. We have shown that the formula of energy associated with these solutions are coincide with that given by Bondi [2].

- We have used the flux of the energy-momentum of gravitational and matter fields [15]. The flux of matter field has no effect since the solutions are vacuum ones to the field equations. The non-vanishing value of the flux of the gravitational energy-momentum indicates the emission of the gravitational waves. 
- Maluf and Faria [15] have applied the flux of the gravitational energy-momentum to a tetrad which is different from that we have obtained here. Our results are in coincident with what obtained before [15].

- Zhang [16] have discussed within the Reimannian geometry the properties of the metric (27). Here we have discussed the gravitational radiation of this metric within the teleparallel geometry by using the flux of the gravitational energy-momentum. Also we have shown that our results are in coincident with that obtained before [16].

- A further work that can be done for the solutions obtained in this work is to study their spatial momentum and angular-momentum. This will be our future work.

\section{Appendix}

Here in this appendix we write the explicitly the calculations used in Eq. (28) and in Eq. (38). For Eq. (28), the non-vanishing components of torsion are

$$
\begin{aligned}
& T^{0}{ }_{01}=-\frac{T^{1}{ }_{01}}{2} \cong-2 \frac{M_{, 0}(u, \theta)}{r}+O\left(\frac{1}{r^{2}}\right), T_{02}^{0} \cong \frac{c_{, \theta}(u, \theta) \sin \theta+2 c(u, \theta) \cos \theta-M_{, \theta}(u, \theta) \sin \theta}{r \sin \theta}+O\left(\frac{1}{r^{2}}\right), \\
& T^{0}{ }_{12}=\frac{-T^{1}{ }_{12}}{2} \cong 2 \frac{M_{, \theta}(u, \theta)}{r}+O\left(\frac{1}{r^{2}}\right), \quad T_{02}^{1}=T_{23}^{3} \cong-\frac{c, \theta(u, \theta) \sin \theta+2 c(u, \theta) \cos \theta}{r \sin \theta}+O\left(\frac{1}{r^{2}}\right), \\
& T_{12}^{2}=T^{3}{ }_{13} \cong \frac{2}{r}+O\left(\frac{1}{r^{2}}\right), \quad T_{03}^{3}=-\frac{c_{, 0}(u, \theta)}{r}+O\left(\frac{1}{r^{2}}\right)
\end{aligned}
$$

and the non-vanishing components of the basic vector are

$$
T^{0} \cong-2 \frac{2+M_{, 0}(u, \theta)}{r}+O\left(\frac{1}{r^{2}}\right), \quad T^{1}=\frac{4+M_{, 0}(u, \theta)}{r}+O\left(\frac{1}{r^{2}}\right)
$$

where

$$
M_{, i}=\frac{\partial M}{\partial x^{i}}
$$

For Eq. (38) the non-vanishing components of the torsion tensor are

$$
\begin{aligned}
& T^{2}{ }_{20}=-T^{3}{ }_{30} \cong-\frac{c, 0}{r}(u, \theta) \\
& T_{30}^{2} \cong-\frac{2 d_{, 0}(u, \theta) \sin \theta}{r}+O\left(\frac{1}{r^{2}}\right), \quad T_{23}^{2} \cong 2 \frac{\left\{d(u, \theta) \cos \theta+d_{, \theta}(u, \theta) \sin \theta\right\}}{r}+O\left(\frac{1}{r^{2}}\right), \quad T_{23}^{3} \cong \frac{r \cos \theta-c_{, \theta}(u, \theta) \sin \theta}{r \sin \theta}+O\left(\frac{1}{r^{2}}\right),
\end{aligned}
$$

and the non-vanishing component of the basic vector is

$$
T^{0} \cong 2 \frac{1+M_{, 0}(u, \theta)}{r}+O\left(\frac{1}{r^{2}}\right)
$$




\section{References}

[1] Shirafuji T and Nashed G G L 1997 Prog. Theor. Phys. 981355.

[2] Bondi H, Van der Burg M G J and Metzner A W K 1962 proc. R. Soc. Londen, Ser. A 269 21; Bondi H 1990 Proc. R. Soc. Lond. A 427249.

[3] Arnowitt R, Deser S and Misner C W 1962 Gravitation: An Introduction to Current Research edited by L. Witten (Wiley, N.Y.).

[4] Ortín T 2004 "Gravity and Strings" Cambridge University Press P. 166.

[5] Møller C 1962 Tetrad Fields and Conservation Laws in General Relativity, Proceedings of the International School of Physics Enrico Fermi, edited by C. Møller (Academic Press, London, 1962); Conservation Laws in the Tetrad Theory of Gravitation, Proceedings of the Conference on Theory of Gravitation, Warszawa and Jablonna 1962 (Gauthier-Villars, Paris, and PWN-Polish Scientific Publishers, Warszawa, 1964) (NORDITA Publications No. 136).

[6] Pellegrini C and Plebański J 1963 Mat. Fys. Scr. Dan. Vid. Selsk. 2 no.4.

[7] Hehl F W 1980 in Proceedings of the 6th School of Cosmology and Gravitation on Spin, Torsion, Rotation and Supergravity, Erice, 1979, edited by P. G. Bergmann and V. de Sabbata (Plenum, New York, 1980); Hehl F W, Neeman Y, Nitsch J and Von der Heyde P 1978 Phys. Lett. B78; Hehl F W, McCrea J D, Mielke E W and Neeman Y 1995 Phys. Rep. 258, 1; Baekler P and Hehl F W 2006 Int. J. Mod. Phys. D15 635.

[8] Chang C C, Nester J M and Chen C M 1999 Phys. Rev. Lett. 83 1897; Tung R S and Nester J M 1999 Phys. Rev. D60 021501; Nester J M and Yo Chin H J 1999 J. Phys. 37 113; Nester J M, Ho F H and Chen C M 2003 Quasilocal Center-of-Mass for Teleparallel Gravity, Proceeding of the 10th. Marcel Grossman Meeting (Rio de Janeiro) gr-qc/0403101; Nester J M 1989 Phys. Lett. A139 112; 1992 J. Math. Phys. 33 910; 1988 Class. Quantum Grav. 51003.

[9] de Andrade V C and Pereira J G 1997 Phys. Rev. D56 4689; de Andrade V C, Guillen L C T and Pereira J G 2000 Phys. Rev. Lett. 84 4533; 2001 Phys. Rev. D64 027502.

[10] Hayashi K 1977 Phys. Lett. 69B 441; Hayashi K and Shirafuji T 1979 Phys. Rev. D19, 3524; 1981 Phys. Rev. D24, 3312; Blagojević M and Vasilić M 1988 Class. Quant. Grav. 5 1241; Kawai T 2000 Phys. Rev. D62 104014; Kawai T, Shibata K and Tanaka I 2000 Prog. Theor. Phys. 104505.

[11] Maluf J W 1994 J. Math. Phys. 35 335; Maluf J W and Kneip A 1997 J. Math. Phys. 38 458; Maluf J W and da Rocha-Neto J F 1999 J. Math. Phys. 40 1490; Maluf J W and Goya A 2001 Class. Quant. Grav. 18 5143; Maluf J W, da Rocha-Neto J F, Toribio T M L and Castello-Branco K H 2002 Phys. Rev. D65 124001; Sousa A A and Maluf J W 2002 Prog. Theor. Phys. 108457.

[12] Maluf J W, Veiga M V O and da Rocha-Neto J F 2007 Gen. Rel. Grav. 39227. 
[13] Dirac P M A 1964 Lectures on Quantum Mechanics (Belfer Gradute School of Science (Monographs Series No. 2) Yeshiva University, New York.

[14] Maluf J W and da Rocha-Neto J F 2001 Phy. Rev. D64 084014.

[15] Maluf J W and Faria F F 2004 Ann. Phys. Leipzig 13604.

[16] Zhang X 2006 Adv. Theor. Math. Phys. 10261. 